Revue de phénoménologie

$27 \mid 2019$

Patočka

\title{
Peindre un nuage. Autoportrait et souci de soi.
}

\section{Benjamin Delmotte}

\section{OpenEdition}

Journals

Édition électronique

URL : https://journals.openedition.org/alter/1927

DOI : $10.4000 /$ alter.1927

ISSN : 2558-7927

\section{Éditeur :}

Association ALTER, Archives Husserl (CNRS-UMR 8547)

\section{Édition imprimée}

Date de publication : 1 novembre 2019

Pagination : 227-246

ISBN : 978-2-9550449-5-7

ISSN : 1249-8947

\section{Référence électronique}

Benjamin Delmotte, "Peindre un nuage. Autoportrait et souci de soi. », Alter [En ligne], 27 | 2019, mis en ligne le 22 décembre 2020, consulté le 13 juin 2021. URL : http://journals.openedition.org/alter/ 1927 ; DOI : https://doi.org/10.4000/alter.1927

Ce document a été généré automatiquement le 13 juin 2021.

Revue Alter 


\title{
Peindre un nuage. Autoportrait et souci de soi.
}

\author{
Benjamin Delmotte
}

1 En cumulant peintures et œuvres graphiques, on a compté ${ }^{1}$ plus d'une centaine d'autoportraits dans l'œuvre de Rembrandt, réalisés sur une longue période, entre 1629 et 1669. Rassemblés, ils constituent en quelque sorte le journal d'un corps et de son vieillissement. Se prendre pour sujet de sa peinture, ce serait ainsi, à travers le temps, témoigner de soi-même, dans l'inconstance même du corps, de l'humeur, du décor, des vêtements et de la lumière.

2 C'est pourquoi, par-delà le cas particulier de Rembrandt, il nous paraît possible de comprendre la pratique de l'autoportrait comme une expression de ce «souci de soimême " (epimeleia heautou) propre à la sagesse delphique et développé dans une part importante de l'histoire de la philosophie. Sa récurrence dans l'œuvre de nombreux artistes en fait l'équivalent de ces exercices spirituels de méditation et de retour à soi notamment recommandés par les stoïciens. Se peindre soi-même serait une manière de se soucier de soi-même, de faire de soi un objet d'étude et d'examen, mais aussi d'inquiétude, et ce, tout au long de sa vie.

3 Le parallèle se soutient d'autant mieux que ce souci de soi - nous y reviendrons en évoquant notamment un célèbre extrait de l'Alcibiade - est souvent envisagé à travers la métaphore de l'œil et en termes de regard sur soi-même. Michel Foucault relie ainsi le souci de soi et l'injonction hellénistique et romaine à la conversion du regard : " Il faut avoir le soi en quelque sorte sous les yeux, sous le regard, il faut l'avoir en vue. Et, de là, toute une série d'expressions comme blepe se (regarde-toi, vous trouvez ça chez MarcAurèle) ou observa te (observe toi toi-même), se respicere (se regarder, retourner le regard vers soi), appliquer son esprit à soi (prosekhein ton noun heauto), etc. Il faut donc avoir le soi devant les yeux $»^{2}$.

4 Bien sûr, le rapprochement entre souci de soi et regard sur soi n'a généralement pas d'autre raison que métaphorique ou comparative. Il n'est toutefois pas anecdotique et le parallèle entre souci de soi et autoportrait mérite selon nous d'être développé pour bien saisir ce qui se joue dans cette pratique picturale. 
5 À cet égard, l'objection qui consisterait à en rejeter la légitimité au motif que le peintre, loin de se livrer à une introspection spirituelle, se perdrait dans l'examen des apparences corporelles, ne nous parait pas pertinente. Au contraire - c'est l'un des enjeux de cette réflexion -, il nous semble que l'autoportrait révèle d'une façon remarquable l'impossibilité de distinguer nettement entre «intérieur» et « extérieur » : l'introspection spirituelle n'est en aucune manière contradictoire de ce que nous appellerions volontiers l'« extraspection» picturale, pour désigner cette façon qu'a le regard du peintre de parcourir les apparences de son corps, de manière à en faire le lieu même de la manifestation du « soi » recherché. Comme toute peinture, l'autoportrait manifeste ainsi selon nous une dimension phénoménologique : il s'agit de dépasser l'opposition du corps et de l'esprit pour découvrir la chair comme «lieu » de la conscience.

$\mathrm{Du}$ reste, ce refus d'opposer totalement le corps et l'esprit et de négliger le vécu corporel n'est pas propre à la phénoménologie et peut déjà se voir chez les stoïciens eux-mêmes. Michel Foucault remarque ainsi à quel point le relevé des détails de santé et de régime est important dans les Lettres de Pline ${ }^{3}$ : le souci de soi n'implique pas nécessairement le mépris du corps, mais au contraire l'attention à la façon dont il nous apparaît et dont nous le vivons.

7 Si tant est, donc, qu'un parallèle est possible entre autoportrait et souci de soi, reste à préciser ce que la peinture apporte de spécifique à la compréhension de l'epimeleia heautou. À cet égard, nous aimerions faire apparaître la caractéristique suivante : le souci de soi-même se confronte d'autant mieux à sa dimension vertigineuse qu'il s'abîme dans l'œil du peintre, et plus généralement dans un regard qui peut être aussi bien celui du peintre que celui du spectateur.

Pourquoi donc le souci de soi serait-il l'occasion d'un vertige ? Et en quoi l'autoportrait manifesterait-il de façon exemplaire pareil vertige?

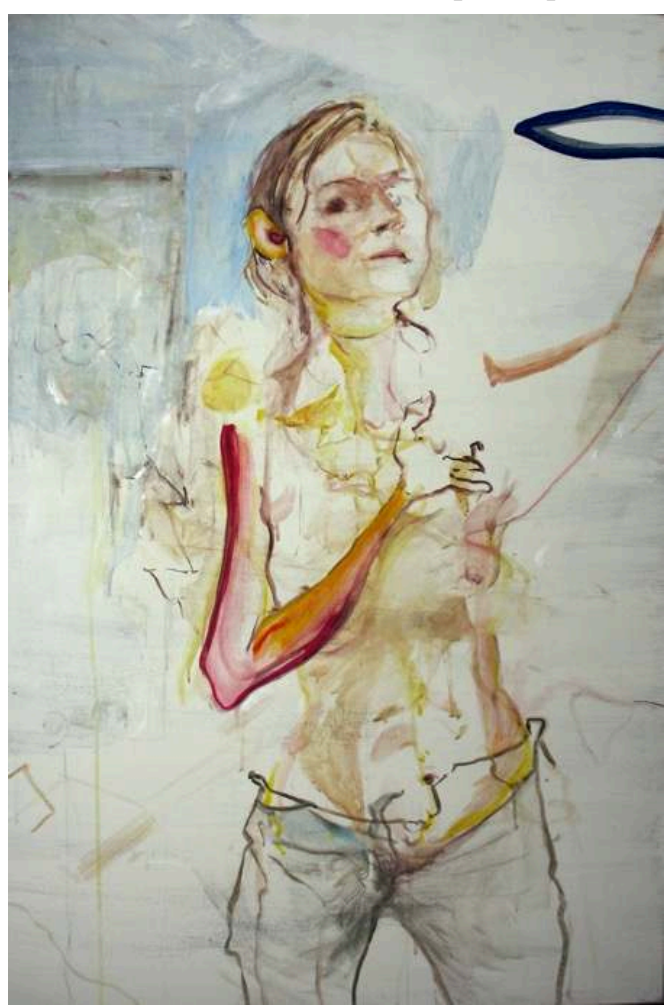


Sans doute le face à face avec soi-même, à travers le vis-à-vis de l'œil et du miroir, est-il l'occasion d'une mise en abyme. Mais par-delà l'effet de ce jeu de miroirs, l'« extraspection » que nous évoquions plus haut renforce le vertige d'un tel vis-à-vis, et demande à être définie plus avant. Le néologisme peut en effet se voir attribuer une utilité conceptuelle intéressante dès lors qu'il désigne non pas seulement un renversement ou une conversion du regard (de manière à trouver l'« intérieur » et même l'intimité dans l'extérieur d'un corps), mais encore une paradoxale éclipse de soi dans le souci de soi-même.

10 À titre méthodologique, nous entendons recentrer notre interrogation sur l'analyse d'un autoportrait particulier, intitulé Peindre un nuage et réalisé en 2008, qui est l'œuvre d'une jeune peintre contemporaine, Ann Loubert ${ }^{4}$. Sans nous interdire de faire référence à d'autres œuvres et artistes, ce recentrage nous paraît nécessaire pour ôter à notre exposé toute prétention historique : l'enjeu est ici de dégager une caractéristique esthétique de l'autoportrait qui nous paraît essentielle, mais non d'examiner sa prégnance et ses variations dans l'histoire de l'art. Annonçons donc d'emblée notre thèse : à travers l'autoportrait, il apparait que le souci de soi-même n'est pas seulement un retour à soi, mais son extrapolation dans le redoublement de soi, l'appel d'un tiers sinon le gouffre de l'autre.

\section{Météorologie du moi}

11 Le titre du tableau d'Ann Loubert peut nous servir d'indice pour caractériser la pratique de l'autoportrait comme une forme de «météorologie du moi». Le peintre se regarde dans le miroir comme on découvre la météo en ouvrant sa fenêtre, il fixe le temps qui passe comme on observe le temps qu'il fait. Ann Loubert le reconnait ellemême : le tableau lui permet de " poser un état de [son] visage, à un moment donné. Hic et nunc $»^{5}$. Insister sur une telle « météorologie » du moi permet d'inscrire le tableau, et la pratique de l'autoportrait en général, dans l'ordre de la contingence et de la particularité. Il correspond à un moment donné, à des circonstances données.

Pour Ann Loubert, la réalisation de Peindre un nuage s'inscrit ainsi dans une temporalité bien précise, il est réalisé lors de sa trentième année et correspond selon elle à des préoccupations et des interrogations particulières à cet "âge pivot»: «Je suis une femme, je n'avais alors pas d'enfants, la question de la féminité et de la sexualité sont posées par devers cet autoportrait. »

13 Cet ancrage temporel et existentiel de l'autoportrait est essentiel, quoique sans doute insuffisant. Comment l'autoportrait, comme souci de soi-même - et donc attention à une certaine permanence extra-circonstancielle -, pourrait-il en effet se réduire aux simples «variations saisonnières » des états du corps et de l'âme? Toujours à suivre Ann Loubert elle-même, cet arrière-plan fondamental (extra-circonstanciel) pourrait notamment se voir dans le fait que le tableau peut prendre la forme d'une vanité : l'artiste ne peint pas seulement le visage d'une femme particulière, âgée de trente ans, mais celui d'un être qui se soucie de l'œuvre du temps et voit poindre le crâne ${ }^{6}$ sous le visage. L'ancrage circonstanciel ne serait ainsi que l'occasion d'un souci de soi plus fondamental: l'autoportrait serait un face-à-face avec la mortalité comme souci, et pourrait ainsi acquérir une dimension plus universelle, à même de toucher tout un chacun, quelles que soient les circonstances. Si donc «météorologie du moi » il y a, 
celle-ci s'inscrit dans une prévision qui serait essentiellement anticipation, découverte de la mort comme possibilité suprême.

Par ailleurs, toujours pour échapper à cette contingence et toucher quelque permanence, peut-être ne faudrait-il considérer les autoportraits que dans une dimension sérielle, de manière à faire apparaître une continuité par-delà les changements secondaires...

C'est ce que l'on voit notamment dans l'œuvre d'une autre jeune artiste contemporaine, Miyuka Schipfer ${ }^{7}$, dont les carnets et l'œuvre en général regorgent d'autoportraits souvent remarquables. Leur contemplation en série est troublante, car elle fait apparaître le regard de la jeune femme comme un absolu au cœur des circonstances. Si le défilé des dessins et peintures fait passer le temps et varier les détails circonstanciés, les yeux fournissent en effet comme deux «points de fixité " dans la succession des images, d'autant plus étranges qu'ils échappent par ailleurs à une définition arrêtée. Jouons de la formule : ces points de fixité n'offrent point de fixité. Ils ne donnent aucun appui. Au contraire, ils agissent comme un piège, un vortex. Ils happent l'attention et l'attirent dans leur abîme.

16 Ces points de fixité semblent donc bien obéir à la définition du point que donne Kandinsky lorsqu'il en fait une union du silence et de la parole ${ }^{8}$. Ces points nous " parlent » dans la mesure où ils semblent offrir un repère à l'attention du spectateur; mais ils nous abandonnent aussi à un silence comparable à celui des espaces infinis que décrivait Pascal ${ }^{9}$. Car ce regard nous échappe, il se retire au plus profond du visage en même temps qu'il s'adresse à nous. Les points de repère restent ainsi paradoxalement insaisissables, à la fois fixes et fuyants, loquaces et silencieux.

Si la dimension sérielle de l'autoportrait a une dimension remarquable dans l'œuvre de nombreux artistes, il ne nous paraît cependant pas nécessaire de contempler une série d'autoportraits pour y voir l'œuvre du souci de soi-même. Observons ce seul tableau d'Ann Loubert pour nous en convaincre : de ce corps à demi-dénudé, du rose aux joues (il faudrait pouvoir dire «à la joue »), nous ne déduisons rien en termes de chaleur ou de température. Si le tableau s'inscrit dans un temps et un lieu donnés, ces derniers se dérobent à toute certitude, comme si le tableau fuyait l'anecdote : on imagine que l'on se situe dans un atelier (puisque l'on croit deviner le coin d'une toile appuyée contre un mur à l'arrière-plan), comme on imagine qu'il est réalisé de jour (la blancheur dominante et les rehauts jaune et rose suggèrent la lumière naturelle et une saison douce). On peut difficilement préciser les choses, et ces simples affirmations restent sujettes à caution.

Le corps qui occupe le centre du tableau est à peine plus défini et demeure plutôt esquissé. Ses contours sont troubles et discontinus. À demi-nu, il semble émerger du pantalon déboutonné, mais malgré sa nudité, le buste reste peu visible, aussi dilué que les couleurs qui le recouvrent. Le tableau trouve certes des zones plus affirmées: le profil gauche ${ }^{10} \mathrm{du}$ visage et le bras gauche replié ont davantage de densité grâce à des contours plus continus, l'utilisation plus affirmée de la couleur et une attention plus soutenue aux détails corporels (cheveux, oreilles, arcade sourcilière...). Rehaussée par des couleurs chaudes (jaune, rose, rouge), cette partie de la toile entre en équilibre avec d'autres, davantage caractérisées par la couleur bleue, et, elles aussi, plus détaillées que le reste du tableau: contour appuyé de ce qu'on imagine être le manche d'un pinceau, bleu du pantalon, arrière-plan bleuté venant auréoler le visage. 
Si météorologie du moi il y a, le temps reste donc incertain. Il est bien difficile de ramener le tableau à une compréhension qui fixerait de façon univoque l'état du corps ou de l'âme ici en jeu. La météorologie du moi peut difficilement passer pour un éclaircissement de la situation. Au contraire, il semble que l'effacement des circonstances permette, par contraste, de déplacer, de recomposer l'expérience, de manière à éliminer toute superficialité et se concentrer sur le souci de soi-même. De même que le tableau Peindre un nuage ne montre pas le nuage ${ }^{11}$ en question, la météorologie du moi se fiche du «temps qu'il fait». Un peu comme ces gens qui semblent ne pas même sentir la pluie ruisseler sur leur visage lorsqu'ils sont accaparés par le souci, la femme qui apparaît sur la toile semble à ce point soucieuse d'elle-même que son environnement et même son corps sont en grande partie déréalisés. Et pourtant - et tout le paradoxe de l'autoportrait réside dans cette nuance - le souci de soi apparaît bel et bien dans ce corps et cet environnement. Si Ann Loubert peint un nuage, c'est donc celui que l'on associe au souci : un nuage passe, une inquiétude naît.

Non seulement le «moi » est-il recentré sur le souci de soi mais encore ce souci est-il recentré sur sa dimension fondamentale. D'un point de vue psychologique, peut-être un souci particulier, nous l'avons vu, motive-t-il la peintre, la pousse-t-il à se représenter à un moment plutôt qu'un autre. Il n'en reste pas moins que ce souci voit fondre toute sa particularité existentielle pour devenir plus essentiel (jusqu'à tendre vers cet existential qu'est l'être-pour-la-mort) et revêtir une forme plus ambivalente, où l'inquiétude le dispute à l'étonnement, sinon à la fascination.

21 Autrement dit, la météorologie du moi ne s'attache aux circonstances, dans leur particularité, que pour y voir l'occasion du souci de soi, dans ce qu'il a de plus fondamental. Ce sera là une des premières déterminations de l'« extraspection » que nous évoquions: le regard attentif à soi-même n'est pas une dissolution dans l'extériorité visible et factuelle (pas plus que dans l'intériorité psychologique). Il se concentre au contraire là où l'extérieur semble déborder sa simple présence circonstancielle pour devenir le lieu même où se manifeste l'epimeleia heautou.

\section{Éclipse du moi et souci de soi-même}

La météorologie du moi a donc le paradoxal pouvoir de perdre ou diluer le moi si ce dernier ne désigne que le «petit moi » comme fait social déterminé et particulier. Le nuage que peint Ann Loubert, c'est peut-être celui qui passe devant ce moi pour mieux l'évacuer. À cet égard, cet autoportrait nous fait penser à une célèbre scène du Horla, lorsque le narrateur croit enfin piéger l'être qui le trouble en se tournant vers un miroir: non seulement celui-ci reste invisible, mais lui-même semble disparaître. Observons en effet la toile pour remarquer que le buste trouble d'Ann Loubert participe de la même étrangeté dans sa visibilité : une brume ou un nuage presque invisible semble passer devant cette poitrine et l'estomper.

Mais cette éclipse ne signifie pas la perte de l'attention à soi. Au contraire, l'attention à soi devient alors réellement souci de soi-même: l'autoportrait est moins la représentation d'un donné, avec toute son assurance objective, que l'inquiétude d'un retrait. Pour le dire autrement, le soi n'apparait que dans le souci - mieux: dans l'apparition de soi-même comme souci ${ }^{12}$. 

noyaux de condensation. Si Ann Loubert peint un nuage, c'est peut-être en ce sens : elle figure la formation d'un "corps-nuage", autour de semblables "noyaux». En l'occurrence, la recomposition/condensation du corps se fait notamment autour de ces « noyaux » que constituent l'œil (qui scrute) et la main (qui peint). Ici, la main gauche (celle qui reste libre) est également remarquable car elle fait un geste qui n'est pas tant de pudeur (couvrir la poitrine dénudée) que retour à soi : ce geste ramène cette femme à elle-même, plus précisément vers cette reconfiguration d'elle-même comme dialogue de l'œil et de la main gauche. Certes, la main qui peint (la main droite) n'apparaît pas à proprement parler, mais elle se confond ici avec le manche du pinceau et l'insistance avec laquelle il est représenté. Pinceau qui n'est que prolongement de la main, main qui prolonge l'œil, œil qui, à son tour, prolonge la main. L'autoportrait comme souci de soi se manifeste donc dans la tension d'une reconfiguration dynamique et multipolaire.

\section{L'ambivalence du regard}

Comment comprendre cette re-concentration du corps dans la bipolarité tendue de l'œil et de la main? Elle est d'autant plus complexe que ces pôles, s'ils constituent des zones de concentration et de densité picturales, semblent également incapables d'acquérir une définition et une assurance parfaites. Comme on l'a vu, la main droite n'apparaît pas à proprement parler; quant à l'œil, la bille ronde de la pupille a beau fournir le principal foyer de rayonnement du tableau, il reste trouble, "nuageux", indéfini, dans sa forme comme dans son expression. Plus qu'un œil - une forme physiologique définie - Ann Loubert peint un regard, dont l'ambivalence est prégnante. Cette ambivalence du regard tient d'abord à la difficulté que l'on éprouve à le caractériser. Sa coloration psychologique est ambiguë (est-ce un regard sérieux ? concentré? un regard de colère ? un regard d'effroi ?) et finalement sans doute secondaire. Il y passe plutôt ce balancement entre «déni » et «défi » dont Pascal Bonafoux ${ }^{13}$ a fait une caractéristique de l'autoportrait.

Comment comprendre ce défi et ce déni ? Sans doute sont-ils ici à entendre en un sens fondamental (en termes heideggériens, il faudrait leur attribuer une valeur existentiale et non existentielle) : ce défi doublé d'un déni, c'est celui d'un regard confronté à l'impossibilité de se voir lui-même. Autrement dit, si le souci de soi-même qui caractérise l'autoportrait se manifeste essentiellement dans la tension du regard, c'est que l'autoportrait constitue le projet impossible d'une "autovision», la vaine recherche d'une réversibilité de la vue qui serait comparable à celle qui est effective dans le toucher. L'autoportrait est l'impossible portrait de l'artiste en "voyant-vu», sur le modèle du « touchant-touché ». 

surface. La tache de couleur qui figure ce regard semble en effet anormalement large et n'a pas de limite nette, comme si ce regard s'étalait en surface. Mais en même temps que l'on constate cet étalement superficiel, ce regard installe un jeu dans la profondeur, lui-même ambivalent, puisqu'on ne sait s'il est de nature centrifuge ou centripète. Le regard est-il tension vers l'extérieur, vers un objet (le reflet dans le miroir) ? Ou regard en dedans, tension vers l'intériorité, introspection? L'alternative semble impossible à tenir, et ses deux termes semblent également soutenables.

31 C'est pourquoi il nous semble ici nécessaire de parler d'extraspection, non pas au sens où le regard serait tendu vers une extériorité définie (un objet, son reflet dans le miroir), mais vers une forme d'extériorité toujours en excès (extra), non identifiable, d'autant moins identifiable que cette extériorité n'est en rien incompatible avec l'intériorité. L'extraspection, encore une fois, n'est pas le contraire de l'introspection: c'est un regard qui remet en question l'opposition de l'intérieur et de l'extérieur, et qui se caractérise avant tout par l'absoluité de sa visée.

\section{La recherche de l'absolu}

Qu'en est-il de cette absoluité propre à l'extraspection? Elle se comprend d'abord comme « extraobjectivité ». À proprement parler, le regard pictural n'est en effet pas porté sur un objet. Ou alors faut-il préciser que cet objet explose et extrapole l'objectivité.

Se regarder, ce n'est pas regarder un simple ob-jet. Ce qui est factuellement posé làdevant (en reflet dans un miroir par exemple), c'est en même temps ce qui est absolument ici, au principe même du regard porté sur ce reflet. Sujet et objet se recouvrent et se distinguent, et cette ambiguïté se retrouve dans la distinction que nous avons déjà évoquée entre l'œil et le regard: l'exactitude objective de la représentation de l'œil ne garantit en rien l'évocation du regard. Au contraire, celui-ci semble déborder sa formalisation objective. Le regard se retrouve moins dans un travail d'objectivation formelle que dans la suggestion dynamique des ambivalences déjà évoquées (surface/profondeur, concentration/étalement, retraite intérieure/tension vers l'extérieur).

Paradoxalement, il faudrait donc dire que le regard ne coïncide avec lui-même que dans une forme de non-coïncidence objective à soi. Et cette «extraobjectivité » serait à mettre en relation avec une forme d'excès dynamique fondamental et rétif à toute formalisation objective.

Cette « extraobjectivité » relève d'autant plus de l'absolu qu'elle peut se comprendre en lien avec l'ici absolu qui commande la relation d'éloignement chez Husserl. Un objet est en effet toujours plus ou moins éloigné de "moi », sans que ce moi puisse lui s'exposer objectivement. Ce « moi » qui regarde l'objet éloigné, remarque Husserl, ne se confond pas totalement avec l'œil ${ }^{14}$, tout en étant au principe du regard. Autrement dit, ce moi qui voit n'est pas vu et s'éclipse du visible justement parce qu'il en est le principe. Il renvoie fondamentalement à la chair, en tant qu'elle se distingue du corps et constitue un centre d'orientation ${ }^{15}$ absolu dans l'espace. Le caractère extraordinaire de l'extraspection à l'œuvre dans l'autoportrait provient selon nous de cette absoluité : à travers un objet visible et inscrit dans l'espace (son propre corps en reflet), le regard du peintre vise le principe même de la visibilité et de l'orientation dans l'espace. Si donc 
l'objectivité est ici débordée, c'est bien par la subjectivité, mais dans son caractère absolu.

Cette tension vers l'absolu qui caractérise l'autoportrait - en tant que souci de soi s'éclairera encore à travers la référence à Platon. Lorsque Socrate recommande à Alcibiade de méditer la célèbre recommandation du temple de Delphes («connais-toi toi-même »), c'est pour l'enjoindre de connaître sa propre âme. À cet effet, il utilise le modèle de la connaissance de l'œil par lui-même pour envisager ce que pourrait être une connaissance de l'âme par elle-même. De même que l'œil se découvre dans le miroir d'un autre œil - plus précisément lorsqu'il regarde sa pupille, soit «ce qu'il y a de meilleur en l'œil $»^{16}-$, l'âme peut se voir dans l'âme d'autrui, plus précisément « en cet endroit où se trouve l'excellence de l'âme ${ }^{17}$ » et qui ressemble « au divin $»^{18}$. Le souci de soi, qu'il soit envisagé dans des perspectives aussi différentes que la dialectique platonicienne ou le travail pictural, s'apparente donc toujours à une recherche de l'absolu, quand bien même cet absolu prend des formes distinctes : la part divine de l'âme chez Platon ; l'ici absolu de la chair qui déborde le corps pour le peintre.

Mais là où il n'y a que rapport d'analogie entre l'œil qui se voit dans un autre œil et l'âme qui se connaît dans une autre âme, une étrange conjointure semble se manifester dans le souci pictural de soi. Car si la chair excède ou déborde le corps, elle se manifeste pourtant en son sein et nulle part ailleurs. Socrate n'envisage la véritable connaissance de soi-même que dans une forme d'élévation et de dépassement du corps (encore une fois, la connaissance de l'œil par l'autre œil n'est qu'une étape ou un indice analogique de ce que pourrait être la connaissance de l'âme par elle-même). Le peintre, au contraire, veut voir dans le corps le lieu même de son débordement absolu. C'est pourquoi l'extraspection dont il fait preuve n'en est que plus complexe et fascinante.

\section{Dédoublement(s)}

En mettant au jour la recherche de l'absolu qui anime le regard du peintre, on touche sans doute à sa caractérisation philosophique la plus fondamentale. Il n'est pas sûr pour autant que cela suffise à déterminer la vérité picturale.

L'impossibilité de se voir (avec la même facilité et la même immédiateté que l'on a à se toucher) impose la médiation d'un dédoublement, et même d'un dédoublement en cascade : l'œil vu redouble celui qui voit ; la surface de la toile redouble celle du miroir, et le reflet dans le miroir redouble lui-même le peintre. Si donc l'autoportrait est une représentation picturale du peintre, il faut, plus que jamais, insister sur le sens du préfixe et y voir l'indice du redoublement et de l'écart qui en résulte.

De la même manière qu'on l'a déjà vue se dédoubler dans le dialogue de l'œil et de la main, Ann Loubert est à la fois ce corps réel tendu dans l'action de peindre, ce reflet qu'elle observe, et cette représentation qui apparait sur la toile. Elle est à la fois ici et là, actrice et spectatrice, dans l'œil qui voit et dans celui qui est vu.

41 Ces dédoublements en cascade font apparaitre toute l'ambiguïté du souci de soi-même, qui implique à la fois différence et répétition. Dans le souci de soi-même, le soi ne peut apparaître simplement : il s'inverse (en raison du miroir), se scinde (l'autoportrait est un portrait de l'artiste en modèle), sinon se diffracte (songeons à l'autoportrait de Johannes Gumpp, qui montre le redoublement du peintre dans son miroir ainsi que 
dans son image sur la toile, tout en offrant une sorte de mise en abyme de la réalisation de l'autoportrait lui-même).

Le soi-même apparait ainsi comme un redoublement de soi (sinon sa démultiplication en cascade), de telle sorte que le trait d'union entre «soi » et «même » est peut-être tout autant un trait de désunion. L'autoportrait impose au soi une sorte de folle assignation locale, un double bind : toi qui es ici, montre-toi là, sois ici dans le là. Cette folie, cette démesure spatiale se retrouve d'ailleurs au niveau temporel, puisque le regard de l'artiste est toujours à la traîne de son propre reflet, et le geste qui prolonge ce regard (la main qui appose la peinture sur la toile) l'est nécessairement davantage encore. Une fois de plus, le paradoxe s'impose : il n'y a de coïncidence à soi que dans la non-coïncidence à soi. En ce sens, le souci de soi-même est bien une inquiétude : celle d'une non congruence à soi-même, d'un très léger décalage, dans le temps comme dans l'espace.

\section{Vanité de la peinture?}

Comment l'autoportrait peut-il conjointement passer pour une recherche de l'absolu $\mathrm{du}$ soi et condamner ce dernier à se perdre dans la structure de redoublement du même ? Comment la recherche de l'absolu peut-elle avoir un sens dans l'autoportrait s'il ne fait que se confronter à l'impossibilité du voyant/vu?

Lorsque Pascal affirmait la vanité de la peinture, c'était pour lui reprocher sa capacité à susciter de l'admiration à travers la simple ressemblance ${ }^{19}$. D'une certaine manière, l'autoportrait pourrait apparaître comme une peinture doublement vaine, puisque la ressemblance qu'il peut offrir semble absolument incapable de prétendre à l'illusion. Si la ressemblance est possible dans un autoportrait, celle-ci ne peut en effet que renoncer au mirage de l'illusion et ne s'affirmer que dans la reconnaissance d'un nécessaire écart.

Il n'est toutefois pas certain que ce constat de vanité soit suffisant pour caractériser l'autoportrait. Au contraire, en manifestant de manière aussi nette la non-congruence à soi, le portrait de l'artiste par lui-même est peut-être ce qui témoigne le mieux du soi. L'autoportrait, comme pratique picturale, permettrait en effet de voir dans le soi non pas la permanence d'une substance, à laquelle il s'agirait de faire retour via l'examen régulier de soi, mais bien plutôt un principe plastique, une dynamique du possible. Comme portrait de soi-même, l'autoportrait arrache le soi à toute forme d'identité constituée, consciente ou simplement latente, et lui offre la possibilité de s'échapper, ne serait-ce que dans une structure de redoublement.

Plutôt, donc, qu'à une vanité de la peinture, l'autoportrait pourrait nous amener à conclure à une sorte d'éthique libertaire de la pratique picturale : elle est ce qui permet au soi de se reconnaître dans son échappée même; mieux : de se connaître en tant qu'échappée, possibilité toujours présente de l'échappée et de la réinvention de soi. En ce sens, on pourrait dire que le redoublement propre au portrait de soi-même est l'indice d'un écart existentialiste par rapport à toute essence.

Cette révélation est d'autant plus remarquable que l'autoportrait est conjointement manifestation de la situation, lucidité de l'engluement dans un corps-objet. Une fois de plus, il faut ici revenir à l'extraspection picturale : le regard du peintre est celui qui se déborde, qui révèle l'échappée dans la situation. Dans son Autoportrait à l'âge de soixante- 
trois ans, Rembrandt semble ainsi constater aussi bien l'empâtement et l'affaissement de son visage que la clairvoyance aigüe d'un regard qui l'en arrache. Nous dirons que l'autoportrait témoigne d'un souci de soi-même qui se confond avec l'étonnement de soi, plus précisément, l'étonnement de ce que l'on pourrait appeler le «soi-autre»: étonnement d'être ce corps affaissé quand on est ce regard aigu ; étonnement d'être ce regard aigu quand on est ce corps affaissé.

\section{Le soi-même comme « soi-autre » : l'appel d'un tiers} signifie cette présence de l'altérité dans le soi-même? À un premier niveau, il nous paraît essentiel de noter à quel point le processus de dédoublement qui caractérise l'autoportrait se prolonge dans l'appel d'un tiers qui n'est autre que le spectateur. À cet égard, il nous faut regarder Peindre un nuage d'une autre manière et remarquer qu'à la tension entre l'œil et la main droite (celle qui peint) s'en ajoute une autre, entre l'œil et la main gauche (celle qui vient recouvrir la poitrine). Cet œil écarquillé comme une bille, combiné à ce qui peut apparaître comme un geste de pudeur, place en effet le spectateur dans une étrange position de voyeur, comme si le regard de la peintre était autant celui, étonné, qu'elle s'adresse à elle-même, que celui, courroucé, qu'elle adresse au spectateur. Le mélange de défi et de déni prend alors une autre coloration, le spectateur étant lui-même défié et dénié dans son voyeurisme. Cet « œil-point » nous surprend et nous gêne comme un reproche silencieux, comme s'il nous intimait la distance et le recul. Remarquons le paradoxe : cet œil nous happe, nous appelle, mais cet appel sonne comme un rappel à l'ordre et une mise à distance.

Ce n'est pas, nous semble-t-il, qu'il faille ici abandonner l'autoportrait à quelque visée moraliste. La relation de voyeurisme ici en jeu déborde largement la situation triviale au sein de laquelle une personne serait surprise dans sa (semi)nudité et lancerait un regard de reproche. Pour le dire très simplement, le voyeurisme n'est évidemment pas factuel, dans la mesure, où, de fait, l'échange de regard entre l'artiste et le spectateur n'est pas réel mais médiatisé par celui qui existe entre l'artiste et son reflet. Ce voyeurisme revêt ainsi une signification plus fondamentale, et relève moins d'une situation morale et sociale que d'un trouble charnel et existential. Ce n'est pas que nous - spectateurs - violons l'intimité d'autrui, mais plutôt que, dans notre altérité même, nous nous sentons appelés à partager un regard, ou plutôt à partager l'ambivalence d'un regard, à entrer dans l'extraspection, dans le dédoublement, tout en sentant l'incongruité - l'impossibilité - de cette « pénétration » du regard.

\section{Un viol du regard}

C'est bien pourquoi le geste de " pudeur » que l'on peut voir dans Peindre un nuage n'est ni anodin ni contrefait, quand bien même il ne s'agit en aucune façon de "raconter " une situation où un personnage serait violé dans son intimité. Le tableau ne raconte pas un tel viol, sans doute parce qu'il est essentiellement ce viol.

51 Le terme peut évidemment surprendre et implique d'être justifié. Là encore, le paradoxe s'impose : ce regard qui relève essentiellement d'une forme de viol ne peut absolument pas en être un. D'abord, il ne saurait y avoir viol au sens propre, parce que 
ce regard est un regard sur soi-même. À quoi il faut ajouter que ce regard se définit par le consentement de l'autre, en un double sens. Ce regard sur soi-même est en effet découverte et accueil de l'étrangeté en soi, mais aussi invitation faite à l'autre (le spectateur) : invitation à partager ce regard, à le prolonger, à s'y (quasi)reconnaître et s'y abîmer.

Le viol du regard que nous voulons voir à l'œuvre dans l'autoportrait est donc de l'ordre de l'impossible. Il faudrait en effet pouvoir penser quelque chose comme un viol de soi-même, notamment parce que le regard, dans l'autoportrait, manifeste une remarquable violence dans sa pénétration.

Cette violence est d'abord de nature phénoménologique: le regard fait exploser la distinction du sujet et de l'objet et force les apparences courantes pour y voir le lieu possible de l'absolu, manifestant ainsi la violence sinon l'ubris de cette pénétration du regard phénoménologique.

Ensuite, le soi s'y découvre autre et comme sous l'effet de l'autre. C'est en effet toujours un regard stupéfié, dont on ressort, comme s'il nous avait entraîné au-delà d'une limite. C'est pourquoi le geste de pudeur, encore une fois, doit se comprendre comme un geste de retour à soi : cette femme pose sa main sur sa poitrine non pas pour se protéger d'un voyeur extérieur, mais comme pour s'assurer qu'elle est toujours là, que c'est bien elle qu'elle considère. Si donc elle se protège du regard de l'autre, cet autre n'est autre qu'elle-même, et le regard qui l'y conduit est comme le regard de l'autre.

Le viol du regard n'est donc pas une violation d'intimité par le regard, mais un viol du regard lui-même, sa quasi substitution dans l'étrange équivalence du soi et de l'autre. Il $\mathrm{y}$ a viol dans la mesure où le regard de l'autre vient se glisser dans celui qu'on porte sur soi-même, sans presque qu'on s'en rende d'abord compte. La substitution s'opère insensiblement et l'on ne s'en émeut que rétroactivement, lorsque la présence de l'autre en soi devient subitement trop grande. L'extraspection désigne alors cette soudaine vision de l'excès de l'autre en soi.

À écrire ces mots, nous revoyons la scène de Baisers volés, dans laquelle le personnage d'Antoine Doinel répète inlassablement son nom en se regardant fixement dans son miroir. La répétition s'emballe et n'a bientôt plus de sens tandis que le regard de JeanPierre Léaud semble se perdre à force de concentration : un nuage vient voiler son regard et pris d'un vertige, le personnage finit par se passer la main sur le visage comme pour se retrouver, alors même qu'il n'aura fait que se regarder et dire son nom. Sans doute se sera-t-il adonné à pareille extraspection et aura-t-il été confronté à pareil viol du regard: dans son propre reflet, le personnage semble avoir vu l'acteur (et réciproquement), sinon le réalisateur qui le filme (là encore avec tous les jeux de réciprocité possibles).

Ajoutons que ce geste de pudeur concerne d'autant plus le spectateur que celui-ci est amené à rejouer pareil "viol ", à se sentir conjointement violeur et violé. Comme spectateur, on se surprend en effet parfois à s'oublier dans l'autre, à entrer dans ce regard pour à la fois l'exercer et le subir, avant de se ressaisir et de s'étonner d'avoir pu à ce point s'être accaparé le regard de l'autre, ce qui est aussi bien avoir été accaparé par le regard de l'autre. Celui qui écrit ces lignes s'est reconnu dans le regard d'Ann Loubert, de Miyuka Schipfer aussi bien que dans celui de Rembrandt ou de Jean-Pierre Léaud. Si une telle impossibilité a pu se réaliser, l'absurdité apparente d'un "viol de soi-même » ne doit peut-être pas nous arrêter. 


\section{L'appel de l'autoportrait} (comme souci de soi-même) implique indéniablement l'empathie. Toutefois, il est sans doute nécessaire de repenser ce concept à l'aune du viol du regard et du silence de l'appel que nous avons mis en évidence. L'empathie, comme tension vers l'autre, doit être « démoralisée » : elle n'est pas nécessairement altruisme, tension vers l'autre, mais une structure existentiale étonnante, où s'indique autant la centralité de l'ego que sa nécessaire ouverture à l'autre.

Nous dirons que l'autoportrait révèle un souci de soi-même qui n'est pas une introspection mais une intropathie, laquelle peut se penser comme l'avers de l'extraspection. 
63 À un premier niveau, la contemplation par le spectateur est empathique dans la mesure où, à travers cette toile peinte, il est amené à (comme) se voir lui-même, comme si le face-à-face avec le tableau reproduisait celui du peintre avec son reflet. Nous nous reconnaissons (quasiment) dans cet œil qui nous fixe, nous ressentons, comme de l'intérieur, sa tension, comme nous reconnaissons, pour la vivre quasi intérieurement, la façon dont le corps semble émerger du pantalon déboutonné. Il nous semble donc pouvoir nous reconnaître dans cette jeune femme que nous ne sommes pas, que nous ne pouvons pas être. Le souci de soi-même gagne là une nouvelle étrangeté : il conduit à se reconnaître, au-delà de l'identité factuelle. Il y a là quelque chose d'assez remarquable: l'autoportrait est en effet une pratique picturale essentiellement marquée par la solitude et l'isolement, mais cette solitude et cet isolement constituent cela même qui peut être partagé.

Ajoutons que cette saisie intropathique de l'autre ne peut pas être une substitution complète. Husserl, dans la dernière Méditations cartésiennes, insiste beaucoup sur l'impossibilité d'une compréhension absolue d'autrui (elle reste analogique et ne se fait que sur le mode du «quasi »). D'une certaine manière, le silence que nous entendons dans l'autoportrait vient manifester cette dimension simplement analogique du soiautre. Entrer dans un autoportrait, ce n'est pas, pour le spectateur, véritablement être l'artiste-modèle: quelque chose nous échappe nécessairement et il apparaît que l'incroyable pouvoir de la vision cul-

mine dans l'audition d'un silence effarant. L'intropathie empathique apparaît ainsi comme une intrusion bien paradoxale, qui nous donne le sentiment de rester au seuil en même temps qu'elle nous fait éprouver la pénétration.

Par ailleurs, à un second niveau, cette empathie est peut-être aussi celle de la peintre pour le reflet qui apparaît devant ses yeux. Toutes les ambiguïtés que nous avons envisagées dans l'empathie éprouvée par le spectateur semblent en effet déjà se jouer dans le cas de l'artiste. À la question «qui peignez-vous dans un autoportrait? », Ann Loubert nous répond: "une femme qui se trouve être moi, dont le visage est changeant, jamais figé, jamais connu une fois pour toutes de fond en comble ${ }^{21} »$. Le portrait de soi-même creuse à ce point l'écart avec soi-même que l'image de soi qui apparaît semble aussi distante que pourrait être celle d'autrui. À cet égard, la peintre est sans doute sa première spectatrice, et le regard qu'elle jette sur sa peinture est aussi empathique - avec toutes les ambiguïtés signalées - que peut l'être celui du spectateur.

Que signifie cette dimension empathique du regard? L'empathie est à comprendre comme une tension d'ici à là : elle consiste à faire du là (le corps qui se dresse face à nous) un quasi ici (comme s'il pouvait être vécu de l'intérieur). L'empathie signifie donc la possibilité de l'accès à l'intimité dans et malgré la distance. La vision empathique peut donc bien se définir comme extraspection, ici au sens d'une quasi introspection, malgré le gouffre de l'autre.

67 À ce titre, l'empathie participe de l'inquiétante étrangeté propre au souci de soi-même : si nous pouvons quasiment nous reconnaître en autrui, le soi-même ne peut que s'abîmer dans le souci comme inquiétude d'un propre qui renvoie au-delà de l'identité factuelle. 


\section{Conclusion : la faveur du tragique}

Loin de constituer simplement un retour à la familiarité heureuse du soi, l'autoportrait est souci de soi-même et confronte autant à l'inquiétante étrangeté de la séparation qu'à l'apaisement étonnant de l'empathie. Les redoublements du même et l'appel d'un tiers amènent en effet l'autoportrait dans l'exacerbation du tragique de la séparation. Peintre et modèle, corps et chair, artiste et spectateur, ici et là... l'autoportrait ne cesse de manifester la séparation. Mais en même temps qu'il est cette manifestation tragique du soi-même, l'autoportrait est la révélation, accueillie avec faveur, du "soi-autre ", notamment à travers la dimension empathique de l'extraspection. L'incapacité à coïncider avec soi-même a ainsi pour corollaire la possibilité d'une (quasi)coïncidence avec l'autre, jusqu'en soi-même. En ce sens, le nuage que peint Ann Loubert n'est sans doute pas de mauvais augure. Au contraire, la tragédie de la subjectivité ouvre la possibilité d'une intersubjectivité tout aussi fondamentale : il ne s'agit que d'apprendre à regarder la tragédie avec faveur.

\section{NOTES}

1. R. Genaille, Rembrandt, Autoportraits, Hazan, Paris, 1963, non paginé.

2. M. Foucault, L'herméneutique du sujet. Cours au Collège de France (1981-1982), EHESS-GallimardSeuil, Paris, 2001, p. 204-205.

3. Ibid., p. 154.

4. Ann Loubert est une artiste de nationalité franco-suisse qui vit et travaille à Strasbourg. On trouvera une présentation de son œuvre sur le site internet suivant: http:// www.annloubert.com.

5. Ce propos d'A. Loubert est extrait de ses réponses à un questionnaire que nous lui avons fait parvenir en 2018.

6. «Il y a une dimension de Vanité très forte dans ce tableau, déjà parce que peignant mon apparence à cet âge-là, j'avais la pleine conscience d'une apparence périssable. Un crâne à peine visible, d'ailleurs tout à fait "caché" se situe à ma droite, donc à gauche du tableau dans une partie bleue. Juste un léger cerne, très léger, je ne voulais pas que cela fût trop explicite. ». Nous citons ici A. Loubert, dans un courrier électronique du 2 août 2018.

7. M. Schipfer est une artiste française qui vit et travaille à Paris. On trouvera une présentation de son œuvre sur le site internet suivant : https://miyukaschipfer.com.

8. «Ainsi le point géométrique est, selon notre conception, l'ultime et unique union du silence et de la parole. [...] le point géométrique a trouvé sa forme matérielle en premier lieu dans l'écriture - il appartient au langage et signifie silence » (Kandinsky, Point et ligne sur plan, Gallimard, Folio essais, Paris, 1991, p. 25).

9. «Quand je considère la petite durée de ma vie absorbée dans l'éternité précédente et suivante, memoria hospitis unius diei praetereuntis, le petit espace que je remplis et même que je vois, abîmé dans l'infinie immensité des espaces que j'ignore et qui m'ignorent, je m'effraie et m'étonne de me voir ici plutôt que là » (Pascal, Pensées (102), Le livre de poche, Classique, Paris, 2000, p. 87-88). 
10. La latéralisation implique de prendre en compte l'inversion spéculaire. Nous avions oublié de le faire dans une première version du texte confiée à l'artiste, qui avait protesté qu'elle n'était pas gauchère! Par-delà l'anecdote, cette erreur nous semble intéressante : elle nous amène à bien nous remémorer l'écart à l'œuvre dans tout autoportrait réalisé devant le miroir. L'image n'est pas imitation, copie, mais nécessaire transformation, modification. En d'autres termes, l'inversion spéculaire nous force à penser à quel point l'autoportrait est l'œuvre de l'autre.

11. Pour A. Loubert, le nuage existe pourtant bien littéralement: «le nuage est bel et bien représenté. Il entre et sort du tableau, sous la forme simplifiée presque enfantine d'un contour bleu foncé, que la main absente, celle qui sort du tableau, esquisse d'un geste que j'imagine (avoir été) rapide et donc fugace, comme un nuage ", A. Loubert, courrier électronique du 2 août 2018.

12. Dans un échange de courrier, $M$. Schipfer nous a fait remarquer que ce lien entre souci et autoportrait était peut-être ce qui permettait de mettre en avant la spécificité de l'autoportrait par rapport au portrait : l'autoportrait ne peut montrer autre chose que le regard soucieux de l'artiste au travail, puisqu'il est ce travail, quand le portrait peut saisir le modèle dans des circonstances très diverses.

13. P. Bonafoux, Les peintres et l'autoportrait, Skira, Genève, 1984, p. 7.

14. «En ce qui concerne l'éloignement, il s'agit ici, objectivement, de l'être-plus-loin ou êtreplus-près d'un objet qui acquiert son second point-de-relation en moi [...]. Plus précisément, ce point-de-relation n'est pas le corps tout entier, il est placé dans la partie de celui-ci qui n'est pas vue, il se trouve quelque part dans la tête, dans l'œil ou derrière l'œil. Du fait qu'il ne tombe pas dans la sphère de l'exposition ni de celle de l'exposable, l'éloignement non plus, comme distance entre l'objet et ce point de relation charnel », Husserl, Chose et espace, Leçons de 1907, trad. par J.-F. Lavigne, \$64, Puf, Épiméthée, Paris, 1989, p. 271.

15. Pour Husserl, le corps-propre se conçoit en effet « en tant que centre d'orientation » («Der Leib als Orientierungszentrum »), Husserl, Idées directrices pour une phénoménologie et une philosophie phénoménologiques pures, Livre second, Recherches phénoménologiques pour la constitution, trad. par É. Escoubas, Puf, collection Épiméthée, 1982, p. 223.

16. Platon, Alcibiade, trad. par C. Marbœuf et J.-F. Pradeau, Flammarion, GF-Flammarion, Paris, 2000, p. 181.

17. Ibid., p.182

18. Idem.

19. "Quelle vanité que la peinture, qui attire l'admiration par la ressemblance des choses dont on n'admire point les originaux !» (Pascal, Pensées (74), op. cit., p. 65).

20. Heidegger, Être et temps, trad. par F. Vezin, Paris, Gallimard, Nrf, 1986, p. 383.

21. Ces propos sont à nouveau tirés des réponses au questionnaire envoyé à l'artiste. 